\title{
Heating Affects Structure, Enterocyte Adsorption and Signalling, As Well as Immunogenicity of the Peanut Allergen Ara h 2
}

\author{
Philipp Starkl ${ }^{1}$, Durga Krishnamurthy ${ }^{1}$, Krisztina Szalai ${ }^{1}$, Ferdinand Felix ${ }^{1}$, Anna Lukschal ${ }^{1}$, \\ Dominik Oberthuer ${ }^{2}$, Hugh A. Sampson ${ }^{3}$, Ines Swoboda ${ }^{1}$, Christian Betzel ${ }^{2}$, Eva Untersmayr ${ }^{1}$, \\ and Erika Jensen-Jarolim ${ }^{1,4}, *$
}

\author{
${ }^{I}$ IPA - Department of Pathophysiology and Allergy Research, Medical University of Vienna, Vienna, Austria \\ ${ }^{2}$ Institute of Biochemistry and Molecular Biology, University of Hamburg, Hamburg, Germany \\ ${ }^{3}$ Department of Pediatrics, Jaffe Food Institute, Mount Sinai School of Medicine, New York, USA \\ ${ }^{4}$ Messerli Research Institute of the Medical University of Vienna and the Veterinary University Vienna, Vienna, Austria
}

\begin{abstract}
Previous studies have indicated that specific molecular properties of proteins may determine their allergenicity. Allergen interaction with epithelia as the first contact site could be decisive for a resulting immune response. We investigate here for the major peanut allergen Ara $\mathrm{h} 2$ whether thermal processing results in structural changes which may impact the protein's molecular interactions with enterocytes, subsequent cellular signalling response, and immunogenicity.

Ara h 2 was heat processed and analyzed in terms of patient IgE binding, structural alterations, interaction with human enterocytes and associated signalling as well as immunogenicity in a food allergy mouse model.

Heating of Ara h 2 led to significantly enhanced binding to Caco-2/TC7 human intestinal epithelial cells. Structural analyses indicated that heating caused persistent structural changes and led to the formation of Ara $\mathrm{h} 2$ oligomers in solution. Heated protein exhibited a significantly higher immunogenic potential in vivo as determined by $\operatorname{IgG}$ and $\operatorname{IgE}$ serum antibody levels as well as IL-2 and IL-6 release by splenocytes. In human Caco-2/TC7 cells, Ara h 2 incubation led to a response in immune- and stress signalling related pathway components at the RNA level, whereas heated allergen induced a stress-response only.

We suggest from this peanut allergen example that food processing may change the molecular immunogenicity and modulate the interaction capacity of food allergens with the intestinal epithelium. Increased binding behaviour to enterocytes and initiation of signalling pathways could trigger the epimmunome and influence the sensitization capacity of food proteins.
\end{abstract}

Keywords: Allergy, food processing, intestinal epithelium, oral mouse immunization, peanut allergen Ara h 2.

\section{INTRODUCTION}

Food allergy is a rapidly spreading worldwide disease. Allergy against peanut (Arachis hypogaea) is one of the most dangerous and frequent type 1 food allergies [1, 2]. Eleven allergens from peanut (Ara h 1-11) have been identified, with Ara h 1 and 2 being of major importance [3]. Recent studies raised evidence that food processing may influence the protein stability in gastric digestion $[4,5]$, scavenger receptor recognition and IgE-binding capacity $[6,7]$.

Previous studies provide evidence that the specific molecular features of allergens may be decisive in the sensitization phase. The molecular properties of the major house dust mite allergens Der $\mathrm{p} 1$ and Der $\mathrm{p} 2$, for instance directly contribute to allergic sensitization $[8,9]$. Ara $\mathrm{h} 1$, one of the major peanut glycoproteins, was found to interact specifi

*Address correspondence to this author at the Department of Pathophysiology and Allergy Research Center of Pathophysiology, Infectiology and Immunology Medical University of Vienna Waehringer Guertel 18-20 1090 Vienna Austria; Tel: +43 140400 5120; Fax: +43 140400 6188;

E-mail: erika.jensen-jarolim@meduniwien.ac.at cally with dendritic cell specific ICAM grabbing nonintegrin (DC-SIGN) [10].

For the initiation of food hypersensitivity we hypothesized that also the interaction of food proteins with the intestinal epithelium as the very first checkpoint upon ingestion could be crucial for the subsequent immune response. Based on the concept of the epimmunome [11], we used Ara h 2 as model allergen and studied its interactions with human intestinal epithelial cells, the subsequent cellular responses as well as its immunogenicity in vivo in a food allergy mouse model. We report here that besides its molecular properties, heating as one of the most important food processing methods influences not only the IgE triggering potential of peanut allergens [12] but also affects the consecutive immune response.

\section{MATERIALS AND METHODOLOGY}

\section{Protein Production}

Coding sequences of Ara h 2 and Cyp c $1[13,14]$ were cloned into a pET26b vector (Novagen, Madison, Wiscon- 
sin) lacking the PelB signal peptide (pET26b plain). PDCD4 was identified as structurally Ara $\mathrm{h} 2$ related control protein using the DALI search engine (http://ekhidna.biocenter.helsinki.fi/dali_server/) [15] and cloned (cDNA clone IMAGp998L1512389Q; Imagenes, Berlin, Germany) into pET26b plain. B121(DE3) (Cyp c 1, PDCD4) and RosettaGami-II(DE3) (Ara h 2) bacteria (both strains from Novagen) were used for recombinant production of $6 \mathrm{x}$ Histagged proteins $[14,16]$ and purified under native conditions using NiNTA superflow columns (Qiagen, Hilden, Germany).

Natural Ara h 2 was purified from roasted or raw, unshelled peanuts (obtained from a local market, originally imported and distributed by Heuschen \& Schrouf, Landgraaf, The Netherlands) as described elsewhere [16].

Allergen purity was determined by SDS-PAGE and subsequent protein staining.

Purified proteins were dialysed against $\mathrm{H}_{2} \mathrm{O}_{\text {dest }}$ and stored at $-80^{\circ} \mathrm{C}$.

\section{Thermal Processing}

Purified proteins were thermally processed in vitro following a protocol previously used to assess the effect of heat treatment and the Maillard reaction on IgE binding from human peanut allergic patients to Ara h 2 [2]. Briefly, proteins were heated at $200 \mu \mathrm{g} / \mathrm{ml}$ in $20 \%$ PBS in Safe Lock tubes (Eppendorf) in a Thermomixer Comfort (Eppendorf) for $90 \mathrm{~min}$ at $99^{\circ} \mathrm{C}$ under shaking. Sample protein content was analyzed by SDS-PAGE/silver staining. For kinetic analyses by circular dichroism analysis, heating was conducted for 5, 10, 15, 30, 45, 60, or 90 minutes.

Before use, frozen Ara h 2 was warmed to $37^{\circ} \mathrm{C}$ and mixed for solution of cryo-precipitates.

\section{Cell Culture}

Human Caco-2/TC7 cells were a kind gift of Monique Rousset (INSERM, Paris, France). Cells were cultured and differentiated as previously described using cell culture plastic from Corning (Amsterdam, The Netherlands) [17].

\section{ELISA Assays}

Allergen specific ELISA for detection of specific antibodies in human or mouse serum was performed as previously described $[18,19]$. Collection of patient sera was approved by the ethics committee of the Medical University of Vienna and performed according to ethical guidelines. Informed consent was obtained from all patients.

For cell ELISA experiments, Caco-2/TC7 cells were differentiated in 96 well flat bottom plates for at least 10 days after confluence. Cells were then kept on ice and washed with cold PBS and blocked $1 \mathrm{~h}$ with $1 \%$ BSA. Next, $50 \mu \mathrm{g}$ recombinant allergen or control protein was added onto the cells in triplicates and incubated on ice for $90 \mathrm{~min}$. Bound protein was detected using biotinylated Penta-His-Conjugate (Qiagen) and HRP conjugated streptavidin (Jackson ImmunoResearch, West Grove, Pennsylvania) and TMB substrate (R\&D Systems, Minneapolis, Minnesota).

\section{Fluorescent allergen binding analysis}

Allergens and control proteins were fluorescently labelled using the DyeLight 488 Labelling Kit (Pierce).

Caco-2/TC7 cells were differentiated in 8 well TissueTek Chamber slides (Nunc) and incubated with $10 \mu \mathrm{g}$ labelled protein for $90 \mathrm{~min}$ on ice, washed, fixed with $3.7 \%$ formaldehyde and nuclear-stained for $30 \mathrm{~min}$ at RT with Hoechst dye followed by mounting. Allergen binding to cells was analyzed by fluorescence microscopy using an Axio Observer.Z1 inverted microscope /AxioVision software (Zeiss, Jena, Germany).

\section{Structural Analysis}

Dynamic light scattering (DLS): DLS measurements were carried out in a MRC Crystallization Plate (96-well SBS format, Swissci AG, Switzerland) sealed with SmartSeal (Greiner, Kremsmünster, Austria) using a Spectro Light 500 plate reader (Molecular Dimensions, Newmarket, United Kingdom) [20]. The Spectro software (Nabitec, Lüneburg, Germany) was used for interpretation of the autocorrelation function [21]. The CONTIN-algorithm [22] was applied to obtain the distribution of particle radii, measurement evaluation, and generation of images.

Circular dichroism (CD) spectroscopy was performed as previously described [14]. The spectra were measured at a scan speed of $50 \mathrm{~nm} / \mathrm{min}$ with a resolution of $0.2 \mathrm{~nm}$. The average of three scans was taken as result after baseline spectrum subtraction. The results are shown as mean residue ellipticity $(\theta)$ at the respective wavelengths.

\section{Mouse Immunization}

Female, 6 weeks old BALB/c mice were obtained from Charles River Laboratories (Willmington, Massachusetts) and treated according to European Union rules of animal care with the permission of the Austrian Ministry of Sciences (BWMF-66.009/01870-II/10b/2009). Mice were orally immunized by intragastric gavage with $500 \mu \mathrm{g}$ of untreated or heated natural Ara h $2(10 \mathrm{mg} / \mathrm{ml})$ in PBS optionally containing $10 \mu \mathrm{g}$ cholera toxin (Sigma) in $100 \mu 1$ total volume (Fig. 3a). Blood samples were weekly taken 1 day prior to immunizations for determination of antigen specific serum antibody levels against the respective immunogen by ELISA. After 6 weeks, immunization was stopped to prevent tolerance induction. One last oral immunization was exhibited 7 days prior to the sacrifice. On day 83, mice were challenged by oral application of $500 \mu \mathrm{g}$ of untreated or heated Ara h 2 and monitored for allergic or anaphylactic symptoms (skin efflorescence, body temperature, activity, diarrhea) for $1 \mathrm{~h}$.

\section{Splenocyte Stimulation}

Splenocyte suspension was seeded at $4 \times 10 \mathrm{e} 5$ cells/well in triplicates in 96 well plates (Corning), and stimulated with plain medium or medium containing $5 \mu \mathrm{g} / \mathrm{ml}$ concanavalin A, $50 \mu \mathrm{g} / \mathrm{ml}$ untreated or heated natural Ara h 2. Supernatant was harvested after $72 \mathrm{~h}$ of incubation and analyzed using anti-mouse IL-2 and IL-6 (eBioscience, San Diego, California) ELISA antibody sets. Cytokine release upon medium stimulation was subtracted as background. 
Table 1. Primer Sequences of Investigated Genes

\begin{tabular}{|c|c|c|c|c|c|c|c|c|c|c|c|c|}
\hline \multirow[b]{2}{*}{$\begin{array}{l}\text { Gene } \\
\text { Symbol }\end{array}$} & \multirow[b]{2}{*}{ Full Name/Synonyms } & \multirow[b]{2}{*}{ NCBI ID } & \multirow[b]{2}{*}{ 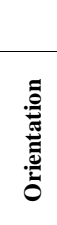 } & \multirow[b]{2}{*}{ Sequence $5^{\prime}$ - 3' } & \multicolumn{8}{|c|}{ Pathway Involvement } \\
\hline & & & & & $\frac{\theta}{Z}$ & 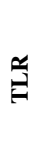 & 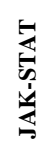 & 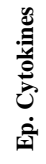 & 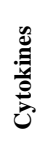 & 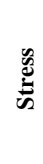 & $\stackrel{\infty}{\check{2}}$ & $\frac{\stackrel{c}{m}}{\stackrel{m}{2}}$ \\
\hline \multirow[t]{2}{*}{ rplpo } & ribosomal protein, large, $\mathrm{P} 0$ & NM_001002.3 & fwd & cctcatatccgggggaatgtg & & & & & & & & \\
\hline & & & rev & gcagcagctggcaccttattg & & & & & & & & \\
\hline \multirow[t]{2}{*}{$c c 120$} & chemokine (C-C motif) ligand 20 & NM_004591 & fwd & gcgaatcagaagcagcaagca & $*$ & & & & & & & \\
\hline & & & rev & tgtgtgaaagatgatagcattgatgtca & & & & & & & & \\
\hline \multirow[t]{2}{*}{$i k b k b$} & $\begin{array}{c}\text { inhibitor of kappa light polypep- } \\
\text { tide }\end{array}$ & NM_001556 & fwd & agatccagatcatgagaaggctga & * & & & & & & & \\
\hline & $\begin{array}{c}\text { gene enhancer in b-cells, kinase } \\
\text { beta }\end{array}$ & & rev & gcagaccacagcagttctca & & & & & & & & \\
\hline \multirow[t]{2}{*}{ tnf } & tumor necrosis factor/TNFa & M10988.1 & fwd & tgacaagcetgtagcccatgt & * & * & & & & & & \\
\hline & & & rev & acctgggagtagatgaggtaca & & & & & & & & \\
\hline \multirow[t]{2}{*}{ ifng } & interferon gamma & NM_000619.2 & fwd & aagagtgtggagaccatcaagga & & * & & & & & & \\
\hline & & & rev & ttggacattcaagtcagttaccgaata & & & & & & & & \\
\hline \multirow[t]{2}{*}{$i l-6$} & interleukin 6 & NM_000600 & fwd & ccctgagaaaggagacatgtaaca & & * & & & $*$ & & & \\
\hline & & & rev & tcctcattgaatccagattggaag & & & & & & & & \\
\hline \multirow[t]{2}{*}{ irf1 } & interferon regulatory factor 1 & NM_002198 & fwd & acctgaggacatcatgaagctcttg & & * & * & & & & & \\
\hline & & & rev & acagctaaagtctccatagacagag & & & & & & & & \\
\hline \multirow[t]{2}{*}{ cxcl9 } & $\begin{array}{c}\text { chemokine (C-X-C motif) ligand } \\
9\end{array}$ & NM_002416 & fwd & tgggagaaacaggtcagccaa & & & * & & & & & \\
\hline & & & rev & tggtgaagtggtctcttatgtagt & & & & & & & & \\
\hline \multirow[t]{2}{*}{ mmp10 } & matrix metallopeptidase $10 /$ & NM_002425 & fwd & tctttgcagttaaagaacatggagact & & & * & & & & & \\
\hline & stromelysin 2 & & rev & gaggaataaattggtgcctgatgc & & & & & & & & \\
\hline \multirow[t]{2}{*}{$n o s 2 a$} & nitric oxide synthase 2 , inducible/ & NM_000625 & fwd & ctacaacatcctggaggaagtg & * & & $*$ & & & & & \\
\hline & iNOS, NOS2 & & rev & gtcacattctgcttctggaaacta & & & & & & & & \\
\hline \multirow[t]{2}{*}{$i l-25$} & interleukin 25 & NM_022789 & fwd & aggtggagcactgtgectgt & & & & * & & & & \\
\hline & & & rev & caagtctctgtccaactcatatctc & & & & & & & & \\
\hline \multirow[t]{2}{*}{$i l-33$} & interleukin 33 & NM_001164724 & fwd & cacattgagcatccaaggaacttca & & & & $*$ & & & & \\
\hline & & & rev & acagattggtcattgtatgtactcag & & & & & & & & \\
\hline \multirow[t]{2}{*}{$t s l p$} & thymic stromal lymphopoietin & NM_033035 & fwd & ctgattacatatatgagtgggaccaaa & & & & $*$ & & & & \\
\hline & & & rev & tgaaggttaggctctggatttcagt & & & & & & & & \\
\hline \multirow[t]{2}{*}{$i l-1 \beta$} & interleukin 1 beta & NM_000576 & fwd & caatcttcattgctcaagtgtctga & & & & & $*$ & & & \\
\hline & & & rev & ctggaaggagcacttcatctgt & & & & & & & & \\
\hline \multirow[t]{2}{*}{$i l-2$} & interleukin 2 & NM_000586 & fwd & atgcccaagaaggccacagaa & * & & & & $*$ & & & \\
\hline & & & rev & actattacgttgatattgctgattaagtc & & & & & & & & \\
\hline \multirow[t]{2}{*}{$i l-8$} & interleukin 8 & NM_000584 & fwd & agctctgtgtgaaggtgcagt & & * & & & $*$ & & & \\
\hline & & & rev & gtccactctcaatcactctcagt & & & & & & & & \\
\hline
\end{tabular}


Table 1. Cont....

\begin{tabular}{|c|c|c|c|c|c|c|c|c|c|c|c|c|}
\hline \multirow[b]{2}{*}{$\begin{array}{c}\text { Gene } \\
\text { Symbol }\end{array}$} & \multirow[b]{2}{*}{ Full Name/Synonyms } & \multirow[b]{2}{*}{ NCBI ID } & \multirow[b]{2}{*}{ 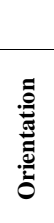 } & \multirow[b]{2}{*}{ Sequence 5' - 3' } & \multicolumn{8}{|c|}{ Pathway Involvement } \\
\hline & & & & & $\frac{\hat{z}}{\bar{\Sigma}}$ & 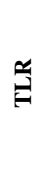 & 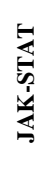 & 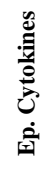 & 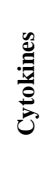 & 总 & $\stackrel{\widetilde{\varkappa}}{\Omega}$ & $\frac{\vec{n}}{\frac{m}{2}}$ \\
\hline$i l-18$ & interleukin 18 & NM_001562 & fwd & ctgattctgactgtagagataatgca & & & & & $*$ & & & \\
\hline \multirow[t]{2}{*}{ fos } & FBJ murine osteosarcoma viral & NM_005252 & fwd & atacactccaagcggagacaga & & & & & & $*$ & & \\
\hline & oncogene homolog & & rev & agctgccaggatgaactctagt & & & & & & & & \\
\hline hsbpl & heat shock factor binding protein & NM_001537 & fwd & tcacctcggtggtgcagaca & & & & & & $*$ & & \\
\hline \multirow[t]{2}{*}{ elk 1} & & NM_001114123 & fwd & ggtactactatgacaagaacatcatc & & & & & & & $*$ & \\
\hline & & & rev & catggtggaggtaacagacac & & & & & & & & \\
\hline \multirow[t]{2}{*}{ map $2 k 3$} & mitogen-activated protein kinase & NM_031168 & fwd & ggagattgctgtgtctatcgtg & & & & & & & $*$ & \\
\hline & kinase 3 & & rev & gcttcacatctctgtggatcac & & & & & & & & \\
\hline \multirow[t]{2}{*}{$b c l 2$} & b-cell CLL/lymphoma 2 & NM_000633 & fwd & ataacggaggctgggatgcctt & & & & & & & & $*$ \\
\hline & & & rev & actgagcagagtcttcagagaca & & & & & & & & \\
\hline$f n 1$ & fibronectin 1 & NM_212482 & fwd & ctggagtctttaccacactgca & & & & & & & & $*$ \\
\hline
\end{tabular}

Overview of primer sequences used for real time PCR experiments. Asterisks in the pathway involvement columns indicate a function of the investigated gene in the respective signalling pathway.

\section{Statistical Analysis}

Statistical comparison between mouse groups was performed by the Mann Whitney $U$ test (two tailed), using GraphPad Prism software (version 5.0 for Windows, GraphPad Software, La Jolla, California). Cell ELISA analysis was conducted using paired t-test (two tailed), gene expression results were analyzed by 1-way ANOVA and Bonferroni post test.

\section{Enterocyte Stimulation and Gene Expression Analysis}

Differentiated Caco-2/TC7 cells were incubated for $1 \mathrm{~h}$, $3 \mathrm{~h}, 6 \mathrm{~h}, 12 \mathrm{~h}, 24 \mathrm{~h}$ or $48 \mathrm{~h}$ in $6-w e l l$ plates with $100 \mu \mathrm{g} / \mathrm{ml}$ untreated or heated Ara h 2 (previously treated with EndoTrap Red [Hyglos, Regensburg, Germany]) or buffer containing LPS (Sigma, \#L2880) equivalent to the protein samples (250pg/ml final concentration). RNA was isolated using the RNeasy Kit (Qiagen) and transcribed into cDNA using the High Capacity cDNA Reverse Transcription Kit (Applied Biosystems, Vienna, Austria). Real-time PCR was conducted on a 7900HT Real-Time PCR System using the Power SYBR Green PCR Master Mix (both Applied Biosystems). Primers (Table 1) span exon/exon boundaries and were checked for specificity using Primer Blast (www.ncbi.nlm.nih.gov/tools/primer-blast). Real-time PCR was conducted in duplicates. $\triangle \mathrm{CT}$ values were calculated based on the expression of the house keeping gene RPLP0 [23]. The relative gene expression levels in cells stimulated with allergen and LPS alone was compared using the $\Delta \Delta \mathrm{CT}$ method. Mean values of three experiments are presented.

\section{RESULTS}

\section{Heating of Ara h 2 Impacts Food Allergen-Enterocyte Interaction}

IgE binding to Ara h 1 and Ara h 2 may increase upon heat associated food processing [2, 4, 12, 24]. ELISA (Fig. 1A) and immunoblots (data not shown) of Ara h 2 using serum pools of peanut-allergic patients indicated that recombinant Ara h 2 acquired a marginally higher IgE-binding capacity upon heating. Mouse programmed cell death protein 4 (PDCD4), a tumor suppressor protein with TLR signalling function was used as structurally homologous (based on an in-silico search) control $[15,25]$. Neither PDCD4 nor the control allergen carp parvalbumin Cyp c 1 were IgE reactive. 

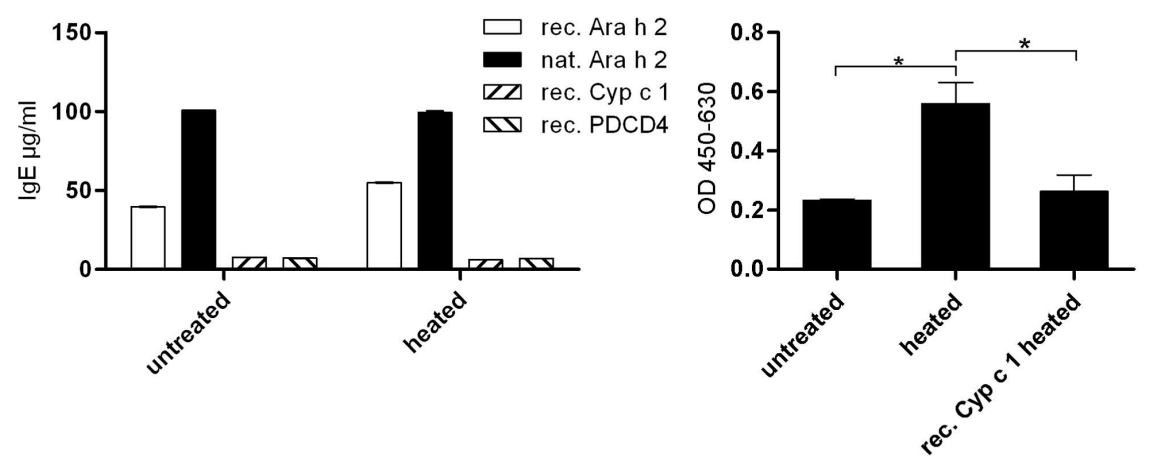

C
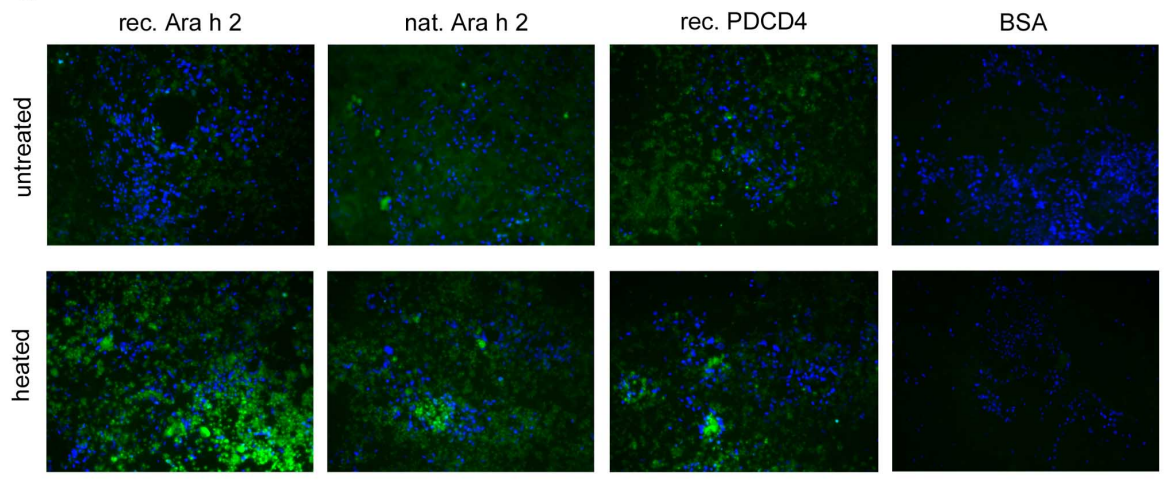

Fig. (1). Heating enhances the adsorption of Ara h 2 to Caco-2/TC7 intestinal epithelial cells rather than IgE binding.

(A) IgE ELISA: Heat processed recombinant Ara h 2, natural Ara h 2, recombinant Cyp c 1 and recombinant PDCD4 were coated on ELISA plates and bound IgE from patient serum pools detected. (B) Human enterocyte allergen binding ELISA: Differentiated human Caco-2/TC7 cells were incubated with recombinant allergens. The amount of bound allergen was detected with anti-His-antibodies. (C) Differentiated Caco-2/TC7 cells were incubated with fluorescently labelled untreated and heated recombinant and natural Ara h 2, recombinant PDCD4 and BSA and the binding pattern and the amount of bound protein (green staining) were visualized by fluorescence microscopy. Stained cell nuclei appear blue. *: P-value $0,01-0,05$;

We attempted to investigate whether heating also influences the adhesion and binding of Ara $h 2$ to intestinal epithelial cells. Differentiated Caco-2/TC7 cells are a suitable model of the human small intestinal epithelial barrier [26]. We incubated these cells with thermally processed recombinant Ara h 2 and observed a significantly higher binding signal with heated Ara h 2 (Fig. 1B).

These data could be verified by fluorescence microscopy analysis of Caco-2/TC7 cells which have been incubated with fluorescently labelled, untreated and heated Ara h 2 or PDCD4 and bovine serum albumin (BSA) as control proteins. Increased binding of heated Ara $\mathrm{h} 2$ in recombinant as well as natural form was observed (Fig. 1C). We also report a slight fluorescence signal with PDCD4 but no detectable signal with BSA. When we tested binding of Ara $h 2$ purified from roasted peanuts to Caco-2/TC7 cells, we did not observe a difference as compared to Ara h 2 from untreated peanuts (data not shown).

\section{Heating Affects Ara h 2 Secondary and Quaternary Structure}

Structural alterations of Ara h 2 caused by heating could be the reason for the increased epithelial adsorption. CD spectroscopy revealed that heating of recombinant as well as natural Ara h 2 had led to conformational changes (Fig. 2).

In contrast to Cyp c 1, Ara h 2 did not refold upon temperature decrease but remained in this partially unfolded state. The irreversible transformation of Ara h 2 secondary structure started after 15 minutes of heating and was mostly accomplished by 45 minutes. Dynamic light scattering (DLS) analysis revealed a significantly increased proportion of protein oligomers in samples containing heated Ara $\mathrm{h} 2$ (Fig. 2).

\section{Heating Increases Ara h 2 Immunogenicity}

Next, we investigated the impact of heat treatment on Ara h 2 immunogenicity in vivo using an oral food allergy mouse model [27]. BALB/c mice were orally immunized with untreated or heated natural Ara h 2 with and without the mucosal $\mathrm{T}_{\mathrm{h}} 2$ adjuvant Cholera toxin (CT). Mice being orally immunized with heated Ara h 2 exhibited increased levels of antigen specific IgG and $\mathrm{IgE}$ levels as compared to feedings with untreated allergen (Fig. 3). CT co-application increased the levels of specific IgG for untreated and heated Ara h 2, but increased IgE only in mice immunized with heated Ara h 2. Oral allergen challenge did not induce significant allergic reactions (data not shown) in any of the groups.

We evaluated a cellular immune response in mouse splenocytes stimulated in vitro with untreated and heated Ara h 2 (Fig. 4). Supernatant of splenocytes from mice immunized with heated Ara h 2 and adjuvant secreted more IL-2 upon 

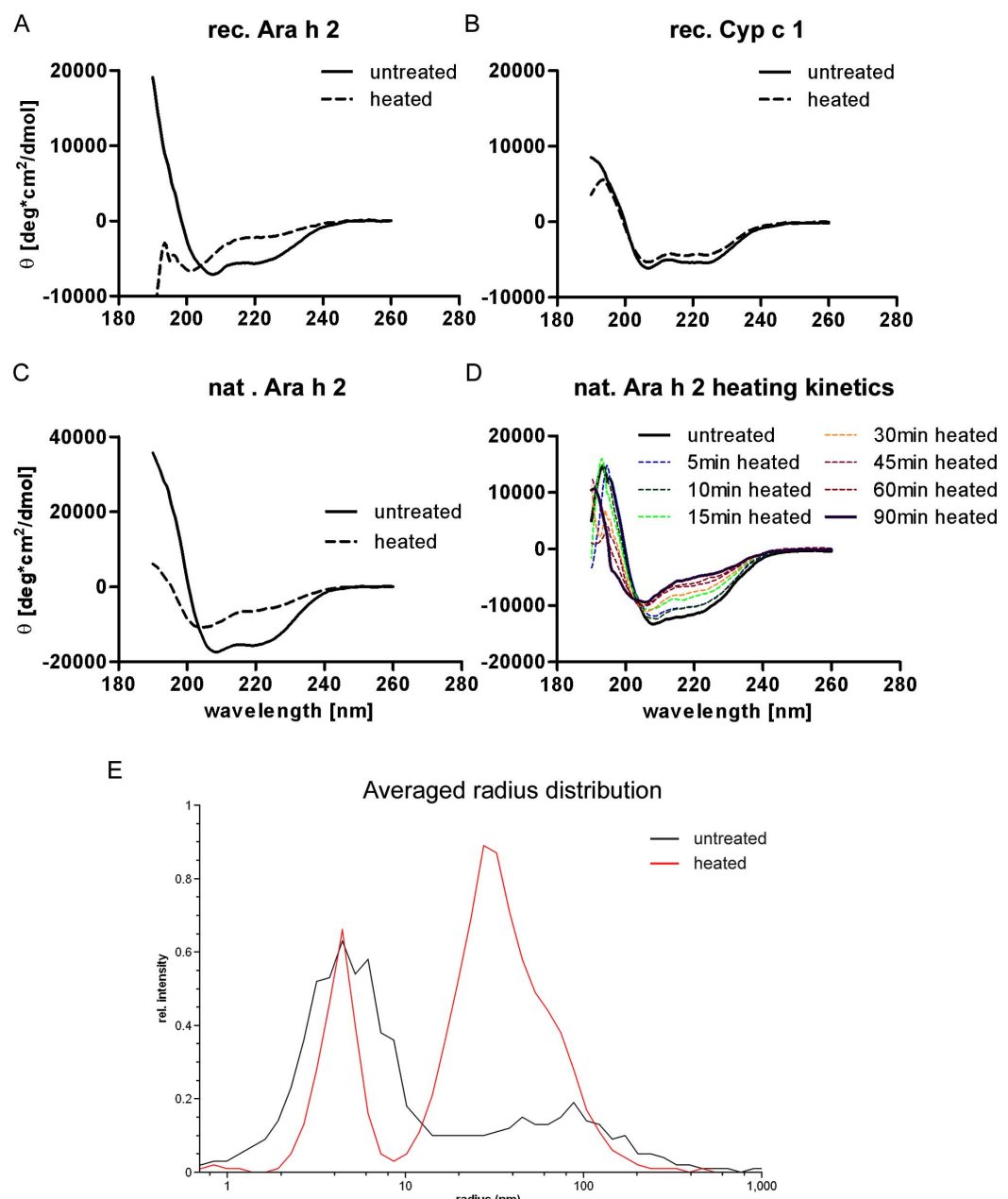

Fig. (2). Heating persistently affects the structure of Ara h 2.

(A-C) CD analysis of heated food allergens: Untreated and heated (C) natural purified and (A) recombinant Ara h 2 and (B) recombinant Cyp c 1 were analyzed by CD spectroscopy. The spectra of untreated (solid lines) and heated (dashed lines) proteins were compared (D) Ara h 2 heat treatment kinetics: Natural purified Ara h 2 was heated for 5-90 minutes. The CD spectra of Ara h 2 after heat treatment were compared with the untreated protein. Untreated (black) and 90min heated (blue) Ara h 2 are represented as solid lines, the other heating time points as coloured dashed lines according to the legend. (E) DLS analysis: The occurrence of protein oligomers of untreated or heated natural Ara h 2 in solution was analyzed by DLS and is depicted as overlay of averaged radius distributions of untreated (green curve) and heated (red curve) Ara h 2.

stimulation with untreated and heated Ara h 2. Similarly, IL6 production was most pronounced in mice treated with heated Ara h 2 and CT.

These in vivo and ex vivo data provide evidence that heat treatment increases the immunogenicity of Ara $h 2$.

\section{Intestinal Epithelial Cells Respond to Ara h 2 Incubation with Altered Gene Expression}

Heat treatment increased the interaction of Ara h 2 with enterocytes (Fig. 1). We hypothesized that the altered allergen-cell contact could also influence a subsequent signalling response. To investigate this idea, Caco-2/TC7 cells were incubated with untreated and heat processed Ara h 2 or buffer containing equivalent Lipopolysaccharide (LPS) amounts. A set of representative genes involved in immunesignalling (NFKB -, TLR-, and JAK-STAT signalling (Fig. 5A-C)) cell differentiation (p38 (Fig. 5G)), Akt/PI3K (Fig. 5H) [28]), cellular stress response (Stress pathway (Fig. $\mathbf{5 F})$ ), and coding for immunomodulatory cytokines $(i l-1 \beta$, il-
18, il-25, il-33, tslp, il-2, il-8 (Fig. 5D, E)) was investigated by real-time PCR. We observed an up-regulation of the $\mathrm{NF} \kappa \mathrm{B}$ signalling component $i k b k b$ after incubation with untreated but not with heated Ara h 2 (Fig. 5A). Both, untreated and heated allergens stimulated the expression of $i l-6$ and irf1 (Fig. 5B). The investigation of genes associated with JAK-STAT signalling associated inducible nitric oxide synthase (nos $2 a$ ) was up-regulated by untreated and heated Ara h 2 (Fig. 5C). Epithelial cell cytokines, $i l-25$, $i l-33$ and $t s l p$ were expressed on a low level (Fig. 5D). Nevertheless, cell contact with both Ara h 2 forms caused an up-regulation of il-33 while untreated Ara h 2 increased TSLP transcription. Inflammatory cytokine genes $i l-1 \beta$ and $i l-18$ were slightly up-regulated in cells treated with either of the allergen preparations (Fig. 5E). Ara h 2, particularly heated allergen, increased expression of the stress related gene fos (Fig. 5F). P38- (Fig. 5G) or Akt/PI3K-signalling components (Fig. 5H) were not influenced. In contrast, Ara h 2 purified from roasted peanuts did not induce a cellular response (data not shown). 
A

$\begin{array}{ll}\text { Oral immunization groups }(\mathbf{n = 8}): & \text { Treatment: } \\ \text { untr. Ara } \mathrm{h} 2 / \text { no } A d j . & \text { o blood withdrawal } \\ \text { untr. Ara } \mathrm{h} 2 /+\mathrm{CT} & \text { * oral immunization } \\ \text { heated Ara h } 2 / \text { no Adj. } & \times \text { intraperitoneal immunization } \\ \text { heated Ara } \mathrm{h} 2 /+\mathrm{CT} & + \text { sacrifice }\end{array}$

CT

Control groups $(n=5)$ :

naive

untr. Ara h 2/IP immunization

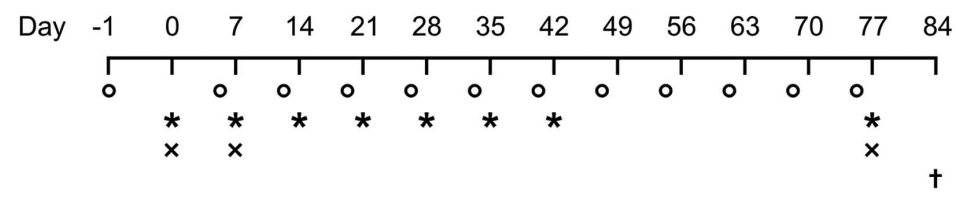

B

C
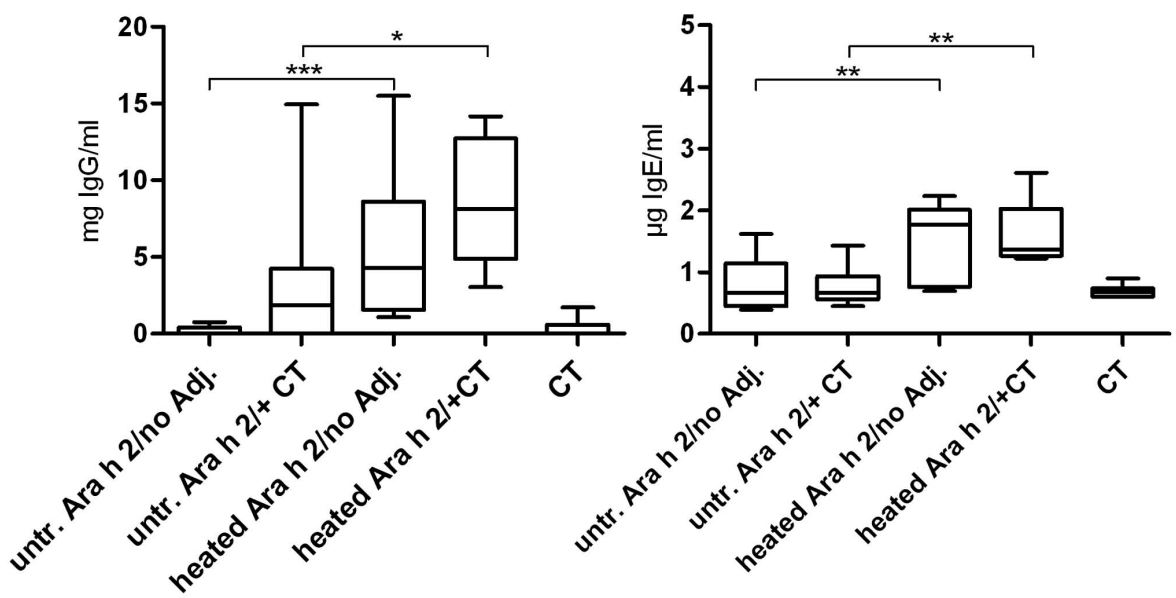

Fig. (3). Oral immunization of BALB/c mice with heated Ara h 2 leads to increased formation of allergen-specific IgG and IgE.

(A) Mouse immunization scheme. BALB/c mice were immunized intraperitoneally (IP) or orally as indicated by intragastric gavage with purified natural Ara h 2 which was either untreated (untr.) or heat-processed. Cholera toxin (CT) was optionally co-applied as mucosal adjuvant (Adj.) in orally immunized groups. (B, C) Serum specificity ELISA: Mice were immunized with untreated or heated natural Ara h 2 alone or in combination with $\mathrm{CT}$ as adjuvant as described and sera were tested for total $\mathrm{IgG}$ and $\operatorname{IgE}$ antibodies specific for the respective immunogen. Sera for the presented (B) IgG and (C) IgE ELISAs were collected 11 weeks after initial immunization. IgG antibody levels of naïve mice were subtracted as background. ***: P-value $\leq 0,001 ; * *$ : P-value $0,001-0,01 ; *$ : P-value $0,01-0,05$;

Our data indicate that Caco-2/TC7 human intestinal epithelial cells may directly and differentially respond to allergen contact at the genomic level.

\section{DISCUSSION}

The factors contributing to food allergy development are only partly understood. In addition to genetic predisposition, environmental factors and structural features of allergenic proteins are of major importance $[5,29,30]$. At the cellular level, important roles during the sensitization phase against food proteins have been ascribed to $\mathrm{T}$ cells, antigen presenting cells as well as to $M$ cells $[31,32]$. A recent study reported a function of intestinal epithelial in sustainment of the allergic state in sensitized individuals [33]. Using the peanut allergen Ara h 2 as model allergen, we here examined the food allergen/enterocyte interaction and investigated whether food processing could influence it. Using a protocol previously applied for testing the effect of heat treatment on Ara $h$ 2-IgE interactions [2], we observed increased adsorption of Ara h 2 to human intestinal epithelial Caco-2/TC7 cells after heat treatment (Fig. 1). Epithelia of the gastro-intestinal tract are in vivo protected by a layer of mucus which prevents the constant stimulation of enterocytes by commensal bacteria and unrestricted access by pathogens. However, the mucus layer is permeable for food proteins as enterocytes may actively take up undigested dietary proteins including food allergens [34, 35].

Structural investigations revealed that heating caused unfolding of the primarily $\alpha$-helical conformation [36] (Fig. 2). This finding is in contrast to previous studies reporting thermo stability of Ara h 2 however, using a gradual, shortterm heating approach [37]. Previous work on roasted Ara h 2 indicated that this processing method was not associated with unfolding [7]. Accordingly, purified roasted Ara h 2 did not show enhanced enterocyte adsorption or affect signalling (discussed below). Further, heating significantly increased the proportion of Ara h 2 aggregates (Fig. 2). Aggregation appears to be a common feature of allergens [38,39]. Milk allergen aggregation upon pasteurization, for instance, contributed to increased allergen uptake from the intestinal lumen and enhanced allergenic potential [32].

Heat treatment also enhanced the immunogenic potential of Ara $\mathrm{h} 2$ in a peanut allergy mouse model (Fig. 3, 4). Pos- 

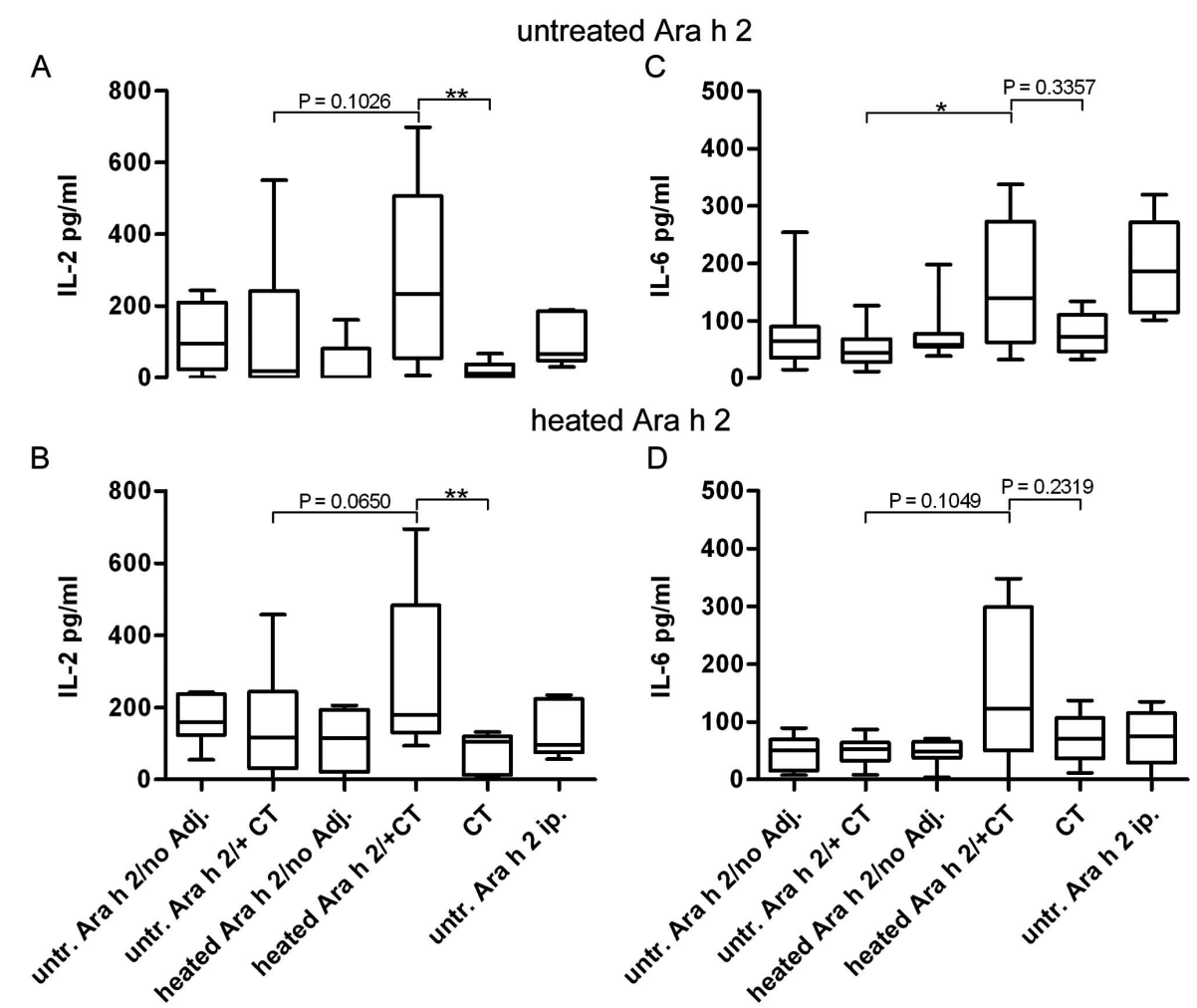

Fig. (4). Splenocytes of mice immunized with heated Ara h 2 release more IL-2 and IL-6.

(A, B) Mouse IL-2 and IL-6 ELISA: Mice were immunized and sacrificed as described. The splenocytes were harvested, seeded in triplicates and stimulated with growth medium containing $(\mathbf{A}, \mathbf{C})$ untreated or $(\mathbf{B}, \mathbf{D})$ heat processed Ara h 2 . The supernatant was screened for $(\mathbf{A}, \mathbf{B})$ IL-2 and (C-D) IL-6 content by ELISA. Background cytokine secretion of cells stimulated with plain growth medium was subtracted. **: Pvalue $0,001-0,01 ; *$ : P-value $0,01-0,05$;

sibly due to the presence of commensal bacteria and intact TLR4 signalling (which is a difference to the originally published model [27]), the $\mathrm{T}_{\mathrm{h}} 2$ bias and IgE levels were not sufficient to cause allergic symptoms or anaphylaxis in any of the groups. We investigated a possible $\mathrm{T}$ cell associated response ex vivo in splenocyte stimulation assays. Heated Ara h 2 caused enhanced secretion of IL-2 and IL-6 by splenocytes originating from mice fed with heated Ara h 2 and CT (Fig. 4).

In contrast to the United States where peanuts are mainly consumed in roasted form, boiling is the most relevant way of peanut processing in Asian countries including China. Despite similar amounts of consumed peanuts, the prevalence to develop peanut allergy is significantly higher in the US [40]. This finding led to the speculation that the way of peanut processing could be the reason for this observation. Indeed, a recent study reported decreased IgE binding to Ara h 2 in extract of boiled and of fried as compared to roasted peanuts [41]. In this context, loss of conformational IgE epitopes associated with the here reported Ara $\mathrm{h} 2$ unfolding upon heating (Fig. 2) could (at least in part) account for this observation. Furthermore, the increased potential of heated Ara $h 2$ to induce a $T_{h} 1$ biased immune response (as concluded from higher specific IgG serum antibody levels (Fig. 3) and from enhanced $T_{h} 1$ cytokine release by spleen cells (Fig. 4) induced by heated Ara h 2) might contribute to the lower incidence of peanut allergy in populations predominantly consuming boiled peanuts $[40,41]$.
Epithelial cells normally have first contact with ingested food allergens. We therefore tested whether Ara h 2 interaction has an impact on the activation state of intestinal epithelial cells. Gene expression analysis revealed that enterocyte contact with Ara h 2 indeed modulated an "epimmunomal response" [11] (Fig. 5): In relation to stimulation with equivalent levels of LPS, Caco-2/TC7 cells exhibited higher expression of NFKB and TLR signalling related $i k b k b, i l-6$, irfl, and nos $2 a$ upon contact with untreated Ara h 2 as compared to heated allergen. An adjuvant effect of natively folded Ara h 2 based on the structural similarity to the TLR signalling component PDCD4 could contribute to the observed cell response. Further, $t s l p$ expression was influenced by untreated allergen. Screening of cytokines revealed a marginal up-regulation of $i l-1 \beta$, il-18, and il-33 in enterocytes upon treatment with both forms of Ara $h$ 2. Heat treated allergen induced higher expression of the stress related gene fos as compared to untreated allergen. These gene expression experiments suggest that $\mathrm{Caco}-2 / \mathrm{TC} 7$ cells respond to the allergen contact with altered expression of genes (ikbkb, irfl, il-6, nos $2 a$, and possibly il-33, tslp, il-1 $\beta$, and $i l-18$ ) associated with immune regulation.

Despite the tendency of enterocytes to support the tolerogenic state against non-dangerous luminal content, they have the potential to produce an array of bioactive molecules which may actively initiate or shape ongoing immune responses [11]. An important example is TSLP which is a major factor of basophil maturation and chemotaxis [42]. TCLP 

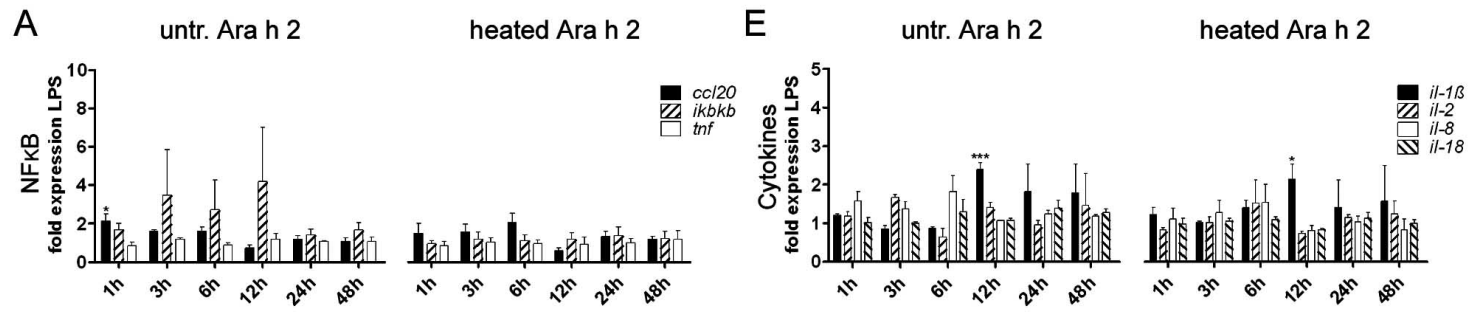

B

$F$

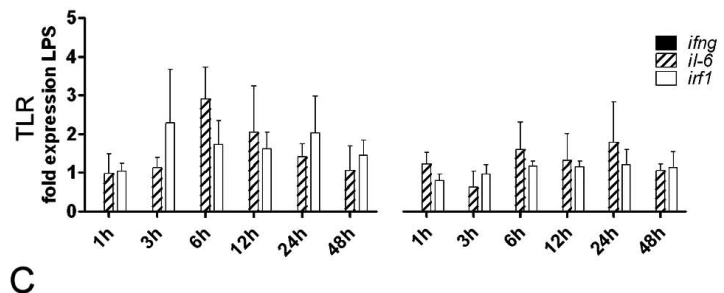

C

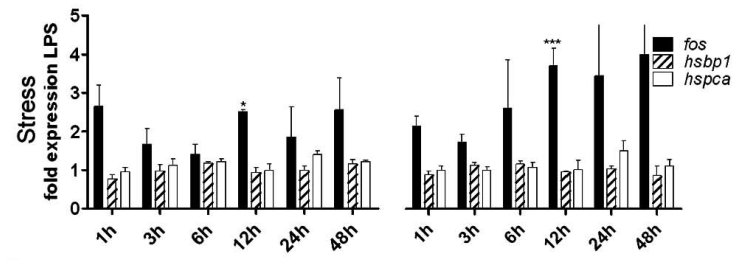

G
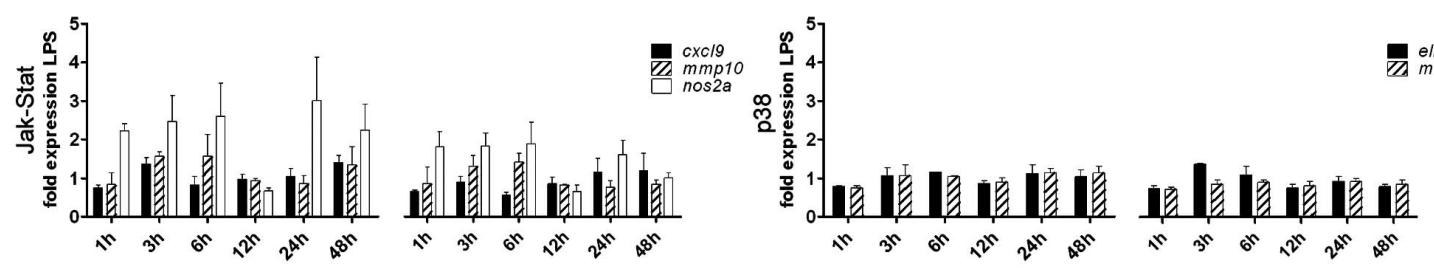

D

$\mathrm{H}$
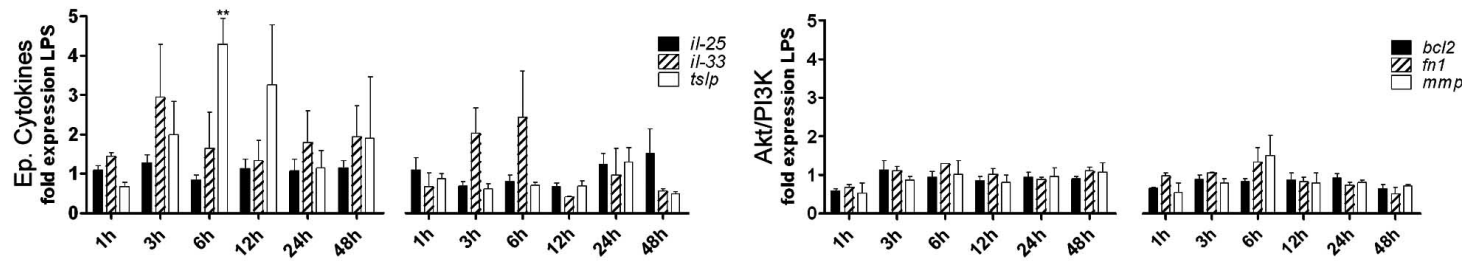

Fig. (5). Intestinal epithelial cells respond to Ara h 2 incubation with differential gene expression.

Gene expression analysis of pathway gene signatures: Caco-2/TC7 cells were stimulated for $1 \mathrm{~h}-48 \mathrm{~h}$ with untreated or heated Ara $\mathrm{h} 2$. The gene expression of representative signalling pathway components of (A) NFkB -, (B) TLR-, (C) JAK-STAT signalling, (D) epithelial cell (Ep.) and (E) pro-inflammatory and T-cell cytokines, $(\mathbf{F})$ stress response, $(\mathbf{G})$ p38- as well as $(\mathbf{H})$ Akt/PI3K signalling (see also Table 1) were analyzed by real-time PCR.

furthermore induces the development of $\mathrm{T}_{\mathrm{h}} 2$ promoting dendritic cells associated with the expression of OX40-ligand [43]. Furthermore, intestinal epithelial cells may secrete chemotactic factors which recruit B- (for instance CCL25, CCL28), Th1- (CXCL9, CXCL10), or $\mathrm{T}_{\mathrm{h}} 2$ cells (CCL1, CCL22) [11]. Certain food proteins might alter the regulation of one or more of these mediators in enterecytes in vivo and therby shape the sub-epithelial immunologic microenvironment and favour the development of hypersensitivity.

To our knowledge, these data represent the first report on a direct response of human enterocytes to food allergen exposure. Although having used the major allergen Ara h 2 as isolated protein, we propose that peanut extract might stimulate intestinal epithelial cells in a similar or even more pronounced way due to the presence of additional immunogenic proteins [3].

\section{CONCLUSION}

Finally, we conclude from our studies that different levels of heating, being important food processing methods, may impact several features related to the immunogenic potential of food proteins, including their direct interaction with intestinal epithelial cells. The subsequently (or not) triggered epimmunomal response might shape the course of allergic sensitization against food proteins as described in a putative basic model (Fig. 6).

\section{CONFLICT OF INTEREST}

All authors declared to have no conflict of interest.

\section{AUTHORS' CONTRIBUTIONS}

Conceived and designed the experiments: PS, DK, EU, EJJ. Performed the experiments: PS, DK, KS, FF, AL, DO. Analyzed the data: PS, DO, IS. Contributed reagents/materials/analysis tools: IS, HAS, CB. Wrote the paper: PS, EJJ.

\section{ACKNOWLEDGEMENTS AND FUNDING}

We thank Tamás Schweighoffer for methodological advices, Diana Mechtcheriakova for the technical support in immunofluorescence microscopy, Karin Stich and Sylvia Knapp for endotoxin measurements, and Stefan Wagner and Christine Hafner for the collection of patient sera. Further, 


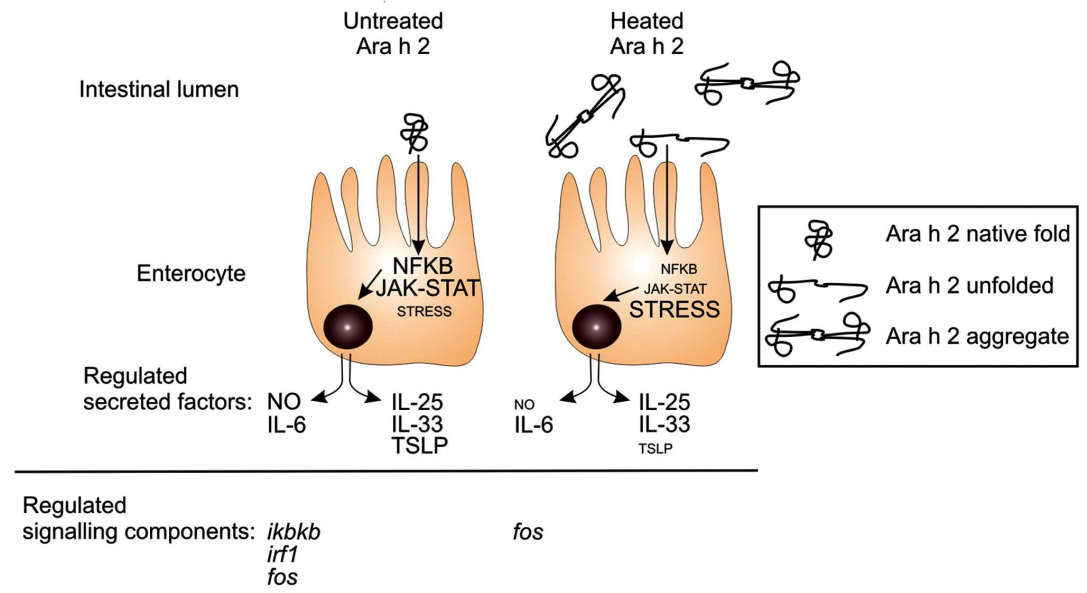

Fig. (6). Putative model of interaction of human enterocytes with the peanut allergen Ara h 2.

Intestinal epithelial cells have earliest contact to food proteins but also different immune stimulatory factors of the gastro-intestinal tract like commensal bacteria. These cells express a broad panel of surface receptors which participate in nutrient uptake and immune sensing. (Left cell scheme) Interaction with the peanut allergen Ara h 2 in its native form leads to initiation of cellular response pathways which finally results in the production of mediators like cytokines and nitric oxide (NO) driving the epimmunome pro-inflammatory. (Right cell scheme) Ara $\mathrm{h} 2$ is persistently defolded and aggregated upon heat treatment affecting its epithelial interaction. Enhanced epithelial binding results in altered signalling and activation state of the cells, primarily inducing a stress-related immune response. Furthermore, the observed increase of aggregates upon heating (see also Fig. 2) could favour another gastro-intestinal uptake route in vivo, via microfold cells for instance [32].

we are grateful to the members of the division 1 at the Department of Pathophysiology and Allergy Research, Oliver Hantschel and Giulio Superti-Furga, (CeMM, Vienna, Austria) and all members of the CCHD doctoral program, particularly Arvand Haschemi, for inspiring discussions.

This work was supported by the doctoral program Cell Communication in Health and Disease (CCHD) of the Austrian Science Fund (FWF), project \#APW01205FW, and Austrian Science Fund project \#SFB F1808-B013. The funders had no role in study design, data collection and analysis, decision to publish or preparation of the manuscript.

\section{REFERENCES}

[1] Burks AW. Peanut allergy. Lancet 2008; 371(9623): 1538-46.

[2] Gruber P, Becker WM, Hofmann T. Influence of the Maillard reaction on the allergenicity of rAra $\mathrm{h} 2$, a recombinant major allergen rom peanut (Arachis hypogaea), its major epitopes, and peanut agglutinin. J Agr Food Chem 2005; 53(6):2289-96.

[3] Finkelman FD. Peanut allergy and anaphylaxis. Curr Opin Immunol 2010; 22(6): 783-8.

[4] Maleki SJ, Chung SY, Champagne ET, Raufman JP. The effects of roasting on the allergenic properties of peanut proteins. J Allergy Clin Immunol 2000; 106(4): 763-8.

[5] Mills EN, Sancho AI, Rigby NM, Jenkins JA, Mackie AR. Impact of food processing on the structural and allergenic properties of food allergens. Mol Nutr Food Res 2009; 53(8): 963-9.

[6] Ilchmann A, Burgdorf S, Scheurer S, et al. Glycation of a food allergen by the Maillard reaction enhances its T-cell immunogenicity: role of macrophage scavenger receptor class A type I and II. J Allergy Clin Immunol 2010;125(1):175-83.

[7] Maleki SJ, Viquez O, Jacks T, Dodo H, Champagne ET, Chung $\mathrm{SY}$, et al. The major peanut allergen, Ara $\mathrm{h} 2$, functions as a trypsin nhibitor, and roasting enhances this function. J Allergy Clin Immunol 2003; 112(1): 190-5.

[8] Trompette A, Divanovic S, Visintin A, et al. Allergenicity resulting from functional mimicry of a Toll-like receptor complex protein. Nature 2009; 457(7229): 585-8.

[9] Wan H, Winton HL, Soeller C, et al. Der p 1 facilitates transepithelial allergen delivery by disruption of tight junctions. J Clin Invest 1999; 104(1): 123-33.

[10] Shreffler WG, Castro RR, Kucuk ZY, et al. The major glycoprotein allergen from Arachis hypogaea, Ara h 1, is a ligand of dendritic cell-specific ICAM-grabbing nonintegrin and acts as a Th2 adjuvant in vitro. J Immunol 2006; 177(6): 3677-85.

[11] Swamy M, Jamora C, Havran W, Hayday A. Epithelial decision makers: in search of the 'epimmunome'. Nat Immunol 2010; 11(8): 656-65.

[12] Maleki SJ, Casillas AM, Kaza U, et al. Differences among heattreated, raw, and commercial peanut extracts by skin testing and immunoblotting. Ann Allergy Asthma Immunol 2010; 105(6): 4517.

[13] Stanley JS, King N, Burks AW, et al. Identification and mutational analysis of the mmunodominant IgE binding epitopes of the major peanut allergen Ara h 2. Arch Biochem Biophys 1997; 342(2): 24453 .

[14] Swoboda I, Bugajska-Schretter A, Verdino P, et al. Recombinant carp parvalbumin, the major cross-reactive fish allergen: a tool for diagnosis and therapy of fish allergy. J Immunol 2002; 168(9): 4576-84.

[15] Holm L, Rosenstrom P. Dali server: conservation mapping in 3D. Nucleic Acids Res 2010; 38 Suppl: W545-W549.

[16] Lehmann K, Hoffmann S, Neudecker P, et al. High-yield expression in Escherichia coli, purification, and characterization of properly folded major peanut allergen Ara h 2. Protein Expr Purif 2003; 31(2): 250-9.

[17] Untersmayr E, Bises G, Starkl P, et al. The high affinity IgE receptor $\mathrm{Fc}$ epsilonRI is expressed by human intestinal epithelial cells. PLoS One 2010; 5(2): e9023.

[18] Untersmayr E, Bakos N, Scholl I, et al. Anti-ulcer drugs promote IgE formation toward dietary antigens in adult patients. FASEB J 2005; 19(6): 656-8.

[19] Brunner R, Wallmann J, Szalai K, et al. Aluminium per se and in the anti-acid drug sucralfate promotes sensitization via the oral route. Allergy 2009; 64(6): 890-7.

[20] Dierks K, Meyer A, Einspahr H, Betzel C. Dynamic Light Scattering in Protein Crystallization Droplets: Adaptations for Analysis and Optimization of Crystallization Processes. Cryst Growth \& Design 2008; 8(5): 1628-34.

[21] Berne BJ, Pecora R. Dynamic Light Scattering. John Wiley \& Sons, Ltd; 1977.

[22] Provencher SW. A constrained regularization method for inverting data represented by linear algebraic or integral equations. Comput Phys Commun 1982; 27(3): 213-27.

[23] Dydensborg AB, Herring E, Auclair J, et al. Normalizing genes for quantitative RT-PCR in differentiating human intestinal epithelial cells and adenocarcinomas of the colon. Am J Physiol Gastrointest Liver Physiol 2006; 290(5): G1067-G74. 
[24] Mondoulet L, Paty E, Drumare MF, et al. Influence of thermal processing on the allergenicity of peanut proteins. J Agric Food Chem 2005; 53(11): 4547-53.

[25] Sheedy FJ, Palsson-McDermott E, Hennessy EJ, et al. Negative regulation of TLR4 via targeting of the proinflammatory tumor suppressor PDCD4 by the microRNA miR-21. Nat Immunol 2010; 11(2): 141-7.

[26] Zucco F, Batto AF, Bises G, et al. An inter-laboratory study to evaluate the effects of medium composition on the differentiation and barrier function of Caco-2 cell lines. Altern Lab Anim 2005; 33(6): 603-18.

[27] Li XM, Serebrisky D, Lee SY, et al. A murine model of peanut anaphylaxis: T- and B-cell esponses to a major peanut allergen mimic human responses. J Allergy Clin Immunol 2000; 106(1 Pt 1): 150-8.

[28] Laprise P, Chailler P, Houde M, et al. Phosphatidylinositol 3kinase controls human intestinal epithelial cell differentiation by promoting adherens junction assembly and $\mathrm{p} 38$ MAPK activation. J Biol Chem 2002; 277(10): 8226-34.

[29] Breiteneder H, Mills EN. Molecular properties of food allergens. J Allergy Clin Immunol 2005; 115(1): 14-23.

[30] Cookson W. The immunogenetics of asthma and eczema: a new focus on the epithelium. Nat Rev Immunol 2004; 4(12): 978-88.

[31] Brandtzaeg P. Food allergy: separating the science from the mythology. Nat Rev Gastroenterol Hepatol 2010; 7(7): 380-400.

[32] Roth-Walter F, Berin MC, Arnaboldi P, et al. Pasteurization of milk proteins promotes allergic sensitization by enhancing uptake through Peyer's patches. Allergy 2008; 63(7): 882-90.

[33] Chen X, Song CH, Liu ZQ, et al. Intestinal epithelial cells express galectin-9 in patients with food allergy that plays a critical role in sustaining allergic status in mouse intestine. Allergy 2011; 66(8): $1038-46$

[34] Menard S, Cerf-Bensussan N, Heyman M. Multiple facets of intestinal permeability and epithelial handling of dietary antigens. Mucosal Immunol 2010; 3(3): 247-59.

[35] Berin MC, Kiliaan AJ, Yang PC, et al. Rapid transepithelial antigen transport in rat jejunum: mpact of sensitization and the hypersensitivity reaction. Gastroenterology 1997; 113(3): 856-64.

[36] Mueller GA, Gosavi RA, Pomes A, et al. Ara h 2: crystal structure and $\operatorname{IgE}$ binding distinguish two subpopulations of peanut allergic patients by epitope diversity. Allergy 2011; 66(7): 878-85

[37] Lehmann K, Schweimer K, Reese G, et al. Structure and stability of $2 \mathrm{~S}$ albumin-type peanut allergens: implications for the severity of peanut allergic reactions. Biochem J 2006; 395(3): 463-72.

[38] Rouvinen J, Janis J, Laukkanen ML, et al. Transient dimers of allergens. PLoS One 2010; 5(2): e9037.

[39] Scholl I, Kalkura N, Shedziankova Y, et al. Dimerization of the major birch pollen allergen Bet v 1 is important for its in vivo IgEcross-linking potential in mice. J Immunol 2005; 175(10): 6645-50.

[40] Hill DJ, Hosking CS, Zhie CY, et al. The frequency of food allergy in Australia and Asia. Environ Toxicol Pharmacol 1997; 4: 101-10.

[41] Beyer K, Morrow E, Li XM, et al. Effects of cooking methods on peanut allergenicity. J Allergy Clin Immunol 2001; 107(6): 107781.

[42] Siracusa MC, Saenz SA, Hill DA, et al. TSLP promotes interleukin-3-independent basophil haematopoiesis and type 2 inflammation. Nature 2011; 477(7363): 229-33.

[43] Zaph C, Troy AE, Taylor BC, et al. Epithelial-cell-intrinsic IKKbeta expression regulates intestinal immune homeostasis. Nature 2007; 446(7135): 552-6.

(C) Starkl et al.; Licensee Bentham Open.

This is an open access article licensed under the terms of the Creative Commons Attribution Non-Commercial License (http://creativecommons.org/licenses/by-nc/3.0/) which permits unrestricted, non-commercial use, distribution and reproduction in any medium, provided the work is properly cited. 